\title{
LA INCLUSIÓN FINANCIERA Y SU INCIDENCIA EN EL INCUMPLIMIENTO TRIBUTARIO DE LAS MYPE PERUANAS (PERSONAS NATURALES)
}

\author{
FINANCIAL INCLUSION AND ITS INCIDENCE ON THE TAX NON-COMPLIANCE \\ OF PERUVIAN MYPE (NATURAL PERSONS)
}

Eloy Tuesta Bardalez Universidad Nacional Pedro Ruiz Gallo Chiclayo, Perú ORCID: https://orcid.org/0000-0003-1224-2093 Correo electrónico: stuesta1977@gmail.com

Rafael Chafloque Gastulo Universidad Nacional Pedro Ruiz Gallo Chiclayo, Perú ORCID: https://orcid.org/0000-0003-1043-6986 Correo electrónico: rchafloqueg@unprg.edu.pe

\section{RESUMEN}

Objetivo: Determinar la incidencia de la inclusión financiera en el incumplimiento tributario de las MYPE peruanas (personas naturales). Método: Se utilizó el método analítico-sintético con un enfoque cuantitativo y un diseño no experimental, considerando como técnica principal el análisis documental y estadístico basado en el modelo de correlación simple de las variables investigativas en un periodo transversal del año 2010 al 2019. Resultados: Los resultados determinan una incidencia directa de la inclusión financiera en el incumplimiento tributario de las MYPE peruanas (personas naturales). Identifica además las brechas de incumplimiento tributario. Conclusión: La inclusión financiera incide de forma directa en el incumplimiento tributario de las MYPE peruanas (personas naturales), motivada por el crecimiento continuo de las brechas de informalidad tributaria en la economía peruana, y dejando en evidencia el desalineamiento e ineficacia de las políticas financieras y tributarias implementadas para promover el desarrollo de las MYPE.

Palabras clave: Inclusión financiera; incumplimiento tributario; informalidad; créditos MYPE; formalización tributaria.

\begin{abstract}
Objective: To determine the incidence of the financial inclusion in the tax non-compliance of Peruvian MYPE (natural persons). Method: The analytical-synthetic method was used, along with a quantitative approach and a non-experimental design, in which the main technique considered was the documentary and statistical analysis based on the simple correlation model of the investigative variables in a cross-sectional period from 2010 to 2019. Results: The results determine a direct incidence in the tax non-compliance of Peruvian MYPE (natural persons). Conclusion: The financial inclusion has a direct impact on the tax non-compliance of Peruvian MYPE (natural persons), motivated by the continuous growth of the tax informality gaps in the Peruvian economy, and revealing the misalignment and ineffectiveness of the financial and tax policies implemented to promote the development of MYPE.
\end{abstract}

Keywords: Financial inclusion; tax noncompliance; informality; MYPE credits; tax formalization.

(c) Los autores. Este artículo es publicado por la revista Quipukamayoc de la Facultad de Ciencias Contables, Universidad Nacional Mayor de San Marcos. Este es un artículo de acceso abierto, distribuido bajo los términos de la licencia Creative Commons Atribución 4.0 Internacional (CC BY 4.0) [https://creativecommons.org/licenses/ by/4.0/deed.es] que permite el uso, distribución y reproducción en cualquier medio, siempre que la obra original sea debidamente citada de su fuente original. 


\section{INTRODUCCIÓN}

El estado del arte de la inclusión financiera y el incumplimiento tributario revela que es un tema sobre el que pocos especialistas han escrito, especialmente en países emergentes como es el caso de Perú (economía con altos índices de informalidad tributaria- laboral). Esto representa para el país un problema histórico y estructural que ha producido diferentes reformas sectoriales con limitados resultados favorables a lo largo de los últimos diez (10) años, siendo aún más relevante para el Estado peruano en una coyuntura de crisis internacional de salud con efectos colaterales causada por la pandemia COVID-19. En el cual se ha intentado cambiar dicha realidad desde la óptica de múltiples reformas legales- tributarias, entre las que destacan las reformas orientadas a las políticas de inclusión financiera y de formalización de las micro y pequeñas empresas peruanas (en adelante MYPE), quienes utilizan créditos de capital de trabajo de las empresas del sistema financiero peruano, tanto personas naturales (formales e informales), como personas jurídicas formales. Dicha inclusión financiera, ha crecido de manera sostenida al igual que los niveles de incumplimiento tributario reflejados en los altos índices de informalidad tributaria- laboral en la economía peruana estudiados en diferentes investigaciones. Frente a la problemática expuesta cabe preguntarse: ¿La inclusión financiera incide en el incumplimiento tributario de las MYPE peruanas (personas naturales)? Asimismo, se plantea como hipótesis general: la inclusión financiera incide de forma directa en el incumplimiento tributario de las MYPE peruanas (personas naturales). La presente investigación tiene por objetivo determinar la incidencia de la inclusión financiera en el incumplimiento tributario de las MYPE peruanas (personas naturales). A continuación, se presenta el estado del arte de las variables investigadas considerando la consistencia metodológica de la presente investigación.

En las principales economías de los países que forman parte de la región Asia Pacífico, se resaltan evidencias de crecimiento del sector financiero en los últimos treinta años, en las cuales se manifiesta su aportación sectorial en el producto bruto interno, en el volumen de activos, rentabilidad de las actividades y la tasa de crecimiento del sector, resaltando la relevancia de contar con un sistema financiero sostenible en el que microempresas, e individuos de bajos ingresos de ambos géneros, accedan a costos financieros competitivos (Girón et al., 2018).

La inclusión financiera involucra la creación, promoción y regulación de un entorno financiero accesible, asequible y seguro para la sociedad, para impulsar el bienestar económico y social con productos y servicios financieros acorde a las necesidades de los diversos sectores de una economía (Carballo y Dalle-Nogare, 2019).
En el ámbito de América Latina, los países que tiene una baja inclusión financiera son Argentina, Perú, Colombia y México, pero en los países de Brasil, Uruguay y Chile se encontraron mejores indicadores del sistema financiero formal en los últimos años, dado que presentan menores índices de informalidad tributaria- laboral, a la par el incumplimiento tributario es relevantes en los demás países (Orazi et al., 2019).

Según Goldenberg (2020), en Chile, las estadísticas a nivel nacional evidencian "la profundidad del mercado financiero y la amplitud creciente de la inclusión financiera, sin embargo, el camino a seguir es continuar perfeccionando los sistemas de protección al consumidor financiero" (p.24), asegurando un mercado financiero seguro, estable y formalizado.

En el caso de Colombia, el gobierno debería concentrar algunos esfuerzos en la lucha contra la informalidad y el incumplimiento tributario, fortaleciendo su Estrategia Nacional de Inclusión Financiera y facilitando el acceso y puntos de contacto con las poblaciones excluidas, para así reducir los costos de los servicios financieros, promoviendo actividades que ayuden a aumentar el entendimiento y la confianza de la población en el sistema financiero, dichas actividades pueden ser de sensibilización y de programas de educación financiera (Cardona Ruiz et al., 2018).

En los últimos dos decenios, se han hecho esfuerzos significativos para modernizar las administraciones tributarias: se han implementado nuevos sistemas informáticos; se han implementado sistemas simplificados para pequeños contribuyentes, racionalizado la estructura de las agencias tributarias; los sistemas de recaudación son ahora más eficientes y el personal cuenta ahora con conocimientos especializados (Organización para la Cooperación y el Desarrollo Económicos [OCDE ], 2020).

Uno de los problemas principales de América Latina es el incumplimiento tributario que se calcula en 335000 millones de dólares (tomando como muestra 16 países en Latinoamérica), considerando los estimados de incumplimiento del impuesto la renta empresarial y el impuesto al valor agregado (IVA), dichos países han adoptado diferentes estrategias de control de las MYPE de los sistemas tributarios y de los grandes grupos económicos vinculados (Comisión Económica para América Latina y el Caribe [CEPAL], 2019).

En Colombia, sobre las conclusiones del estudio vinculado a la pobreza, recursos e informalidad, se resalta el papel del Estado. Las empresas, tanto privadas como públicas y también la misma sociedad, deben de reconocer el desarrollo sostenible como requisito para equilibrar las tres dimensiones, las cuales son: social, ambiental y económica. 
Para equilibrar las tres dimensiones se pueden establecer acuerdos democráticos que induzcan a realizar cambios institucionales y den comienzo a reglas explícitas inclusivas (leyes, políticas públicas, códigos, contratos, y derechos), buscando un equilibrio entre la riqueza e ingresos del Estado (Lara Rodríguez et al., 2019).

La administración tributaria colombiana (DIAN) tiene poca capacidad para controlar la evasión y la elusión, y existen grandes brechas de informalidad donde una gran cantidad de medianos y pequeños empresarios que obtienen incluso altas rentabilidades operan informalmente para evadir impuestos (Espitia et al., 2017).

La inclusión financiera, como se definió previamente, involucra la creación, promoción y regulación de un entorno financiero accesible, asequible y seguro para la sociedad, para impulsar el bienestar económico y social con productos y servicios financieros acorde a las necesidades de los diversos sectores de una economía. En el caso de Perú, en lo que concierne a la demanda, el contexto es competitivo y abierto, no existen topes de tasas de interés de los diversos tipos de créditos y se permiten operaciones de agentes no bancarios (Carballo y Dalle-Nogare, 2019).

El sistema financiero peruano ofrece múltiples servicios, tanto a las empresas MYPE como a los hogares y otras entidades a través de la Banca Múltiple, Cajas Municipales y Rurales de Ahorro y Crédito, Entidades para el Desarrollo de las Micro y Pequeña Empresa, Cooperativas, otras entidades no reguladas como las Organizaciones No Gubernamentales (ONG) y múltiples canales informales de financiamiento. La información disponible revela que las colocaciones para las MYPE han experimentado una expansión continua pero carente de una efectiva regulación que promueva su desarrollo y formalización (León, 2018).

El Instituto Nacional de Estadística e Informática (INEI, 2019), informa que al finalizar el año 2018 operaban en la economía peruana más de 8.5 unidades productivas, con un alto índice de informalidad que supera el $80 \%$. Dicho problema de informalidad permanece durante décadas en la economía peruana sostenida en diversas causas, lo que evidencia un nivel alto y continuo de incumplimiento tributario de las MYPE peruanas.

Los productos financieros en el Perú son aprovechados por un número limitado de empresas (37.6\%) cita los resultados de la Encuesta Nacional de Empresas de 2015 (ENE) (Ministerio de Economía y Finanzas [MEF], 2018b).

En su investigación sobre la inclusión financiera y el incumplimiento tributario de las de MYPE, Lahura (2016) concluye determinando una estimación de 1840554 personas naturales como informales con créditos MYPE; es decir, son clientes del sistema financiero pues a pesar de incumplir las normas tributarias, han contratado créditos con entidades del sistema financiero peruano (estima un 19\% de la cantidad de créditos MYPE), esta investigación evidencia que el incumplimiento no es una barrera legal para contratar un crédito MYPE en el sistema financiero peruano.

Zamalloa et al. (2017) analizan las políticas de inclusión financiera en el tejido empresarial peruano, y concluyen que las mismas deberían comprender y analizar la realidad del mercado formal, debido a que no conlleva una mejoría en el uso de servicios financieros, comenta: "el Estado debe facilitar el proceso de inclusión financiera en el país, asimismo, debe acompañar a los agentes para que éstos puedan migrar naturalmente a servicios financieros formales y supervisados" (p.124), en otras palabras, dar mayores oportunidad a los agentes informales.

El presente artículo busca determinar la incidencia de la inclusión financiera en el financiera en el incumplimiento tributario de las MYPE peruanas (personas naturales), para lo cual basa su análisis en el método analítico- sintético con un enfoque cuantitativo, bajo un diseño no experimental. Se utilizó como técnica principal el análisis documental y el modelo estadístico de regresión lineal simple de las variables investigadas, en función a la información cuantitativa publicada por entidades estatales como el Banco Central de Reserva del Perú (BCR), el Ministerio de Economía y Finanzas (MEF), el Instituto Nacional de Estadística e Informática (INEI), la Superintendencia de Banca y Seguros (SBS) y la Superintendencia Nacional de Aduanas y de Administración Tributaria (SUNAT), en un periodo transversal del año 2010 al 2019.

La estructura del artículo comprende los antecedentes internacionales y nacionales del tema investigado; en el segundo apartado la metodología basada en el análisis estadístico con un enfoque cuantitativo de las variables investigadas; el tercer apartado, los resultados analizados y la discusión correspondiente.

Considerando la normatividad competente, las MYPE se clasifican de la siguiente manera:

La Tabla 1 explica la clasificación de los tipos de MYPE peruanas en función al nivel de ventas (ingresos) anuales, tal como se ha establecido en las normas tributarias - laborales peruanas, pudiendo observar que las microempresas tienen ventas anuales hasta 150 UIT que equivale a $S / 630,000$ soles o \$186,391 dólares. Con respecto a las pequeñas empresas, ellas tienen ventas anuales hasta 1,700 UIT que equivale a S/ $7,140,000$ o \$2,112,426 dólares. Finalmente, con las medianas empresas, tienen ventas hasta 2,300 UIT que equivale a S/ 9,660,000 soles o \$2,857,988 dólares. 
Tabla 1

Clasificación de las MYPE peruanas

\begin{tabular}{ccc}
\hline Tipo de MYPE & Ventas Año & UIT Año 2019 \\
(S/ 4,200 USD 1,303) & S/ 630,000 USD 195,450 \\
Microempresa & 150 UIT & S/ 7,140,000 USD 2,215,100 \\
Pequeña Empresa & 1,700 UIT & S/ 9,660,000 USD 2,996,900 \\
Mediana Empresa & 2,300 UIT & U U
\end{tabular}

Nota: Según lo establecido en la Ley N. ${ }^{\circ}$ Ley 30056- Tipo de cambio promedio. 3.38(S/ por USD).

Fuente: Elaboración propia

Acorde con los alcances de la Ley del Registro de contribuyentes de la SUNAT (Decreto Legislativo $\mathrm{N}^{\circ} .943$, 2003), con repecto a la obligación tributaria establece en el artículo $2^{\circ}$ que: "Deben inscribirse en el RUC a cargo de la SUNAT, todas las personas naturales o jurídicas que sean contribuyentes y/o responsables de tributos administrados por la SUNAT, conforme a las leyes vigentes" (p.1).

El mencionado Decreto Legislativo establece que las micro y pequeñas empresas que ejercen actividad económica en el Perú están obligadas a formalizarse en los registros de la SUNAT, considerando los regímenes tributarios y los supuestos normativos del Código tributario vigente (Decreto Supremo N. ${ }^{\circ} 133-2003-E F, 2003$ ), debiendo cumplir con las demás obligaciones formales y sustanciales del régimen elegido, el hecho de no hacerlo genera el incumplimiento tributario.

La reforma de las políticas de Inclusión Financiera en proceso de implementación del Estado peruano (Decreto Supremo N. $\left.{ }^{\circ} 255-2019-E F, 2019\right)$ valora la importancia de promover en el país:

Las herramientas promotoras de la inclusión social y el desarrollo económico han venido cobrando relevancia a nivel mundial. Así, diversos organismos internacionales, como organismos internacionales como son el Banco Mundial, el FMI, la Alliance for Financial Inclusión (AFI), la Organización para la Cooperación y el Desarrollo Económico (OCDE), el Grupo de los 20 (G20), entre otros. (p.6)

\section{MATERIALES Y MÉTODOS}

La presente investigación tuvo un enfoque cuantitativo que consistió en el análisis estadístico de información pública materializada en tablas, figuras y el modelo estadístico de correlación simple, utilizando para su estudio el método analítico-sintético, basado en la técnica del análisis documental de la información publicada de los años analizado (2010 al 2019).
En un inicio, se tuvo acceso a información pública secundaria de créditos de negocios otorgados a las MYPE peruanas publicada por la SBS y de personas naturales con negocio formales (registrados en la SUNAT). Se determinaron por años la cantidad total de personas naturales con acceso a créditos MYPE y la cantidad de personas naturales informales (que representan el incumplimiento tributario) estimadas por una diferencia simple entre la cantidad de inscritos en el RUC de la SUNAT y los reportados por la SBS con acceso a créditos MYPE. Información descrita en las tablas presentadas en el apartado de resultados.

En una segunda etapa, se analizó la información de las tablas a fin de estimar la correlación lineal Pearson entre las dos (02) variables investigadas en diferentes escenarios, determinando una correlación positiva y significativa entre la totalidad de personas naturales con créditos MYPE y la brecha estimada de incumplimiento tributario de personas naturales con créditos MYPE, en los años 2010 al 2019. Asimismo, se proyectó tres (03) escenarios de personas naturales formales con créditos MYPE (el análisis parte de un escenario base: el 100\% de personas naturales formales cuentan por lo menos con un crédito MYPE en el sistema financiero).

\section{RESULTADOS}

La Tabla 2 muestra en las primeras columnas las cifras del Producto Bruto Interno (PBI) de la economía peruana (Banco Central de Reserva del Perú [BCRP], 2020) y la presión tributaria reportada (MEF, 2018a), evidenciando con un análisis simple una relación no consistente ni directa, no reporta un indicador competitivo (a mayor crecimiento del PBI mayor crecimiento de la Presgión Tributaria). Al cierre del año 2019 la presión tributaria registra un indicador del (14.2\%), cifra menor a la registrada en el año 2010 (15,3\%), lo que representa una presión tributaria por debajo del promedio de países de América Latina (34.2\%), reportada en el informe multianual 2020-2023 (MEF, 2019). 
Tabla 2

Cifras económicas y tributarias MYPE peruanas

\begin{tabular}{ccccccc}
\hline Año & PBI (mills.US\$) & $\begin{array}{c}\text { Presión Tribu- } \\
\text { taria }\end{array}$ & $\begin{array}{c}\text { MYPE } \\
\text { (INEI- miles) }\end{array}$ & $\begin{array}{c}\text { MYPE Formales } \\
\text { (miles) }\end{array}$ & $\begin{array}{c}\text { MYPE Formales } \\
\text { (PN-miles) }\end{array}$ & \% Informalidad \\
\hline 2010 & 149,036 & $15.3 \%$ & 7,922 & 1,222 & 1,035 & $85 \%$ \\
2011 & 171,146 & $16.0 \%$ & 7,826 & 1,344 & 1,131 & $83 \%$ \\
2012 & 193,130 & $16.5 \%$ & 7,831 & 1,495 & 1,250 & $81 \%$ \\
2013 & 202,107 & $16.3 \%$ & 7,653 & 1,606 & 1,347 & $79 \%$ \\
2014 & 202,342 & $16.5 \%$ & 7,700 & 1,714 & 1,436 & $78 \%$ \\
2015 & 191,323 & $14.7 \%$ & 7,570 & 1,817 & 1,528 & $76 \%$ \\
2016 & 194,660 & $13.5 \%$ & 8,017 & 1,930 & 1,624 & $76 \%$ \\
2017 & 214,352 & $12.9 \%$ & 8,245 & 2,011 & 1,759 & $76 \%$ \\
2018 & 225,308 & $14.1 \%$ & 8,502 & 2,212 & 1,867 & $74 \%$ \\
2019 & 230,809 & $14.2 \%$ & 8,767 & 2,410 & 1,995 & $73 \%$ \\
\hline
\end{tabular}

Nota: La cantidad de MYPE del INEI del año 2019 se estimó en base al porcentaje de crecimiento de los años (2018/2017). Los datos de la presión tributaria, la cantidad total de MYPE, Personas Naturales (PN) se tomó en cuenta la información publicitada por la SUNAT en su sitio web.

Fuente: Elaborada con información del Banco Central de Reserva del Perú (BCRP,2021) (PBI), Superintendencia Nacional de Aduanas y de Administración Tributaria (SUNAT,2021) y el Instituto Nacional de Estadística e Informática (INEI,2019).

Tabla 3

Brechas de incumplimiento tributario - MYPE (personas naturales)

\begin{tabular}{cccccc}
\hline Año & $\begin{array}{c}\text { MYPE } \\
\text { con créditos } \\
\text { (PNC) }\end{array}$ & $\begin{array}{c}\text { MYPE } \\
\text { Formales (PNF) }\end{array}$ & $\begin{array}{c}\text { Brecha de } \\
\text { Incumplimiento } \\
\text { (PNC-100\% PNF) }\end{array}$ & $\begin{array}{c}\text { Brecha de } \\
\text { Incumplimiento } \\
\text { (PNC-50\%PNF) }\end{array}$ & $\begin{array}{c}\text { Brecha de } \\
\text { Incumplimiento } \\
\text { PNC-30\%PNF) }\end{array}$ \\
\hline 2010 & 1,578 & 1,035 & 543 & 789 & 1,105 \\
2011 & 1,791 & 1,131 & 660 & 896 & 1,254 \\
2012 & 1,887 & 1,250 & 637 & 944 & 1,321 \\
2013 & 1,919 & 1,347 & 572 & 960 & 1,343 \\
2014 & 1,978 & 1,436 & 542 & 989 & 1,385 \\
2015 & 2,002 & 1,528 & 474 & 1,001 & 1,401 \\
2016 & 2,164 & 1,624 & 540 & 1,082 & 1,515 \\
2017 & 2,346 & 1,759 & 587 & 1,173 & 1,642 \\
2018 & 2,519 & 1,867 & 652 & 1,260 & 1,793 \\
2019 & 2,723 & 1,995 & 728 & 1,362 & 1,906 \\
\hline
\end{tabular}

Fuente: Elaborada con información de créditos de la SBS y la información de Personas Naturales (PN) publicada por la SUNAT en su sitio web.

Asimismo, la Tabla 2 evidencia una reducción de los estimados de informalidad tributaria (forma parte sustancial del incumplimiento tributario), pudiéndose observar que en el periodo analizado (de 2010 a 2019) se disminuyó en un 12\%; es decir al cierre del año 2019, permanece un alto índice de incumplimiento tributario, es decir, de cada 100 MYPE que operan en la economía peruana como personas naturales 73 son informales, lo que afecta de forma directa el empleo formal y otros indicadores de la economía peruana, como un problema permanente del sistema tributario lo que afecta la presión tributaria y lo convierte como un sistema poco predecible dependiendo su recaudación de un limitado número de contribuyentes concentrados en el sector minero y otros servicios (Tuesta y Espinoza, 2020).

La Tabla 3 muestra las MYPE-Personas Naturales con Créditos (PNC) en el Sistema Financiero peruano, regulada por 
la (Superintendencia de Banca y Seguros [SBS], 2020) y la información de Personas Naturales Formales (PgNF) inscritas en el RUC de la Administración Tributaria (SUNAT, 2020).

Asimismo, la Tabla 3 estima el cálculo de las brechas de incumplimiento tributario para lo cual se plantea tres (03) escenarios, una brecha asumiendo que el $100 \%$ de personas naturales MYPE inscritos en el RUC de la SUNAT cuentan con un crédito en el sistema financiero, un siguiente que asume un 50\% con créditos MYPE y un último $30 \%$. El escenario que más se asemejaría a la realidad económica de las MYPE es el último (30\% MYPE con créditos y formales), evidenciado en el estudio del Ministerio de Economía y Finanzas tomado como referencia los estudios de los organismos competentes (MEF, 2018b). La primera brecha estimada de informalidad tributaria al cierre del año 2019 resulta en: 1 millón 906 mil personas naturales MYPE que incumplen las normas tributarias, es decir, que cuentan con créditos de empresa en el sistema financiero peruano, cuya información está siendo protegida de forma no adecuada por el secreto bancario amparado en las normas constitucionales del sistema jurídico peruano, en el sentido que no ha sido analizada ni utilizada por los entes competentes para implementar políticas financieras-tributarias.

La Figura 1 muestra el cálculo de las correlación de las variables investigadas bajo el modelo estadístico de Correlación Simple, entre la variable Personas Naturales con Créditos MYPE (PNC) y la Brecha de Incumplimiento Tributario (PNC-100\% PNF), siendo la misma positiva y directa (coeficiente de correlación: 0.6887 ) con una tendencia de crecimiento, es decir, a mayor cantidad de créditos MYPE otorgados por el sistema financiero mayor es la brecha de incumplimiento tributario a lo largo de los últimos diez (10) años analizados, lo que no contribuye a la formalización tributaria de las MYPE - Personas naturales. El presente resultado nos permite confirmar la hipótesis general planteada en la presente investigación, es decir, la inclusión financiera incide de forma directa en el incumplimiento tributario de las MYPE peruanas (personas naturales).

\section{DISCUSIÓN}

Los resultados evidencian el crecimiento sostenido de los créditos MYPE en la economía peruana, otorgados a empresas formales e informales por diversas entidades reguladas y no reguladas por la SBS. Habiendo crecido las brechas de incumplimiento tributario, dichos resultados coinciden con los alcanzados por las investigaciones de León (2018), el Instituto Nacional de Estadística e Informática, (2019), Lahura (2016) y Zamalloa et al. (2017), dejando en evidencia las debilidades de las políticas de inclusión financiera y de formalización tributaria del Estado peruano.

Las políticas de inclusión financiera de todos los sectores de la economía peruana no están alineados a las políticas

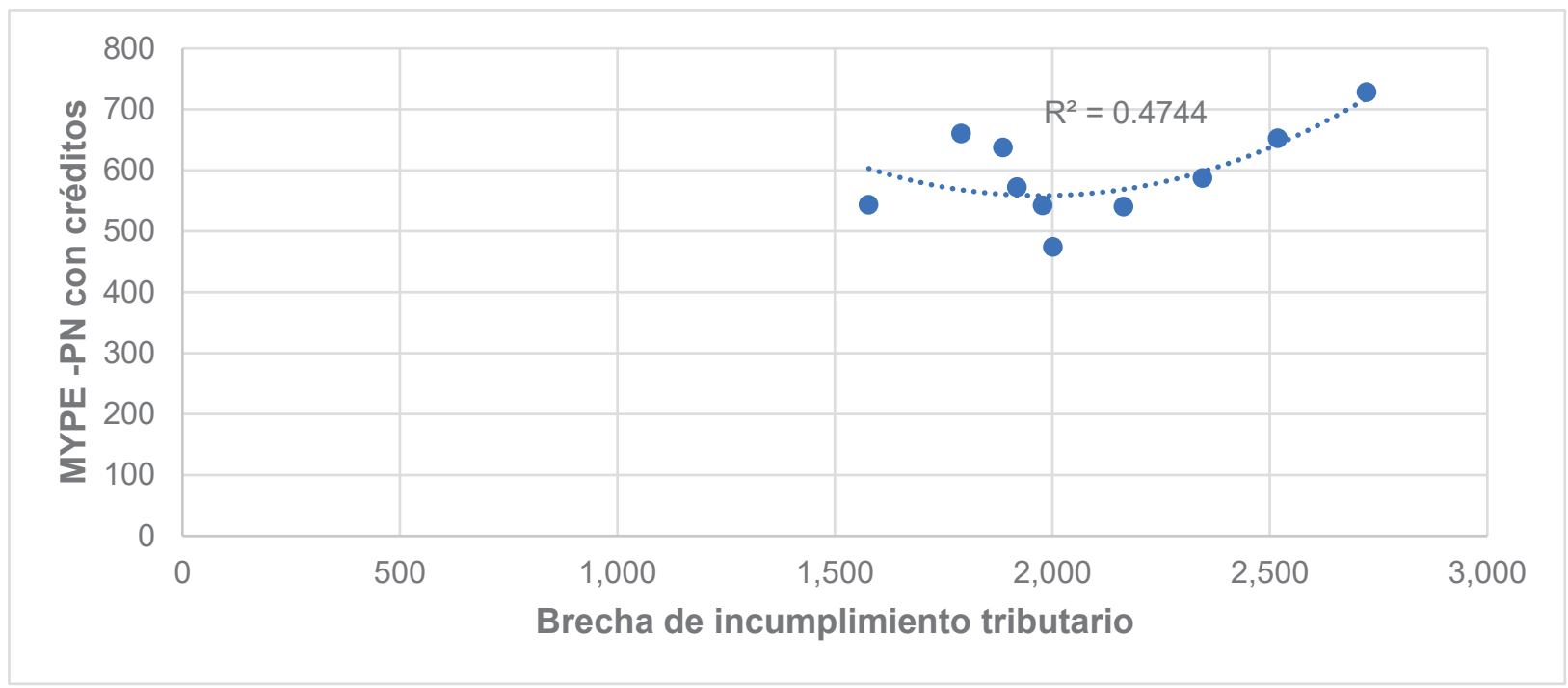

Figura 1. Regresión lineal de variables investigadas

Nota: Coeficiente de correlación: 0.6887- Software SPSS

Fuente: Elaboración propia 
de promoción y formalización tributaria, lo que afecta la presión tributaria del sistema, siendo relevante tener en consideración el alineamiento con los resultados publicados por entidades internacionales como la Comisión Económica para América Latina y el Caribe (CEPAL, 2019) y la Organización para la Cooperación y el Desarrollo Económico (OCDE, 2020).

La inclusión financiera tiene una relación directa con el incumplimiento tributario de las MYPE (personas naturales), evidenciada por el crecimiento continuo de las brechas de informalidad tributaria en la economía peruana, y representa una brecha estimada superior al 1.5 millones de personas naturales con créditos MYPE sin inscripción en el registro de la Superintendencia Nacional de Aduanas y de Administración Tributaria (SUNAT) al cierre del año 2019. Esta informalidad dificulta actualmente la implementación de estrategias efectivas contra el COVID-19 por parte del Estado peruano, cuyo efecto directo es la ampliación de la brecha de incumplimiento tributario en los diferentes sectores productivos de las MYPE. Sin embargo, a la vez representa una gran oportunidad para enganchar a las MYPE en su rescate con la posibilidad de apoyo del Estado y la rebaja de las tasas de interés ofrecidas por las empresas del sistema financiero, por efecto de reducción de riesgo al tener la oportunidad de ser parte de la formalización tributaria y al percibir beneficios directos.

Una de las principales causas del problema del incumplimiento tributario de las MYPE en el sistema tributario peruano, obedece al divorcio entre las políticas de inclusión financiera y las diferentes políticas tributarias implementadas orientadas a promover el desarrollo de las MYPE, representando un vacío normativo perjudicial para el cumplimiento de las obligaciones tributarias (la no obligación de inscribirse en el registro de la Administración Tributaria para obtener un crédito MYPE como persona natural). Asimismo, evidencia el limitado desarrollo de la investigación en temas estratégicos y operativos por parte de la Superintendencia Nacional de Aduanas y de Administración Tributaria (SUNAT) central y de otros entes competentes del sistema como los Ministerios competentes.

Se evidencia la necesidad de cambios normativos y operativos en el sistema tributario a efecto de lograr los estándares exigidos por la OCDE, siendo de las más relevantes las vinculadas con la inclusión financiera formalizada para MYPE, debiendo evaluarse la derogación del secreto bancario a fin de lograr la implementación de políticas financieras-tributarias transparentes y competitivas, aprovechando las grandes ventajas de simplicidad que facilitan las Tecnologías de Información y Comunicación (TIC) del entorno y teniendo en cuenta además la presencia de organismos no regulados por la SBS que otorgan créditos en la economía peruana como las Organizaciones No Gubernamentales (ONG), las
Cooperativas y el mercado informal de prestamistas con intereses usureros muy superiores a las tasas promedio del mercado financiero regulado. Parte de esta regulación se ha concretado con los alcances del Decreto Supremo N..$^{\circ} 430$ 2020-EF (2020) que regula el suministro de información financiera a la SUNAT para combatir la evasión y elusión tributaria (Incumplimiento Tributario).

Urge la implementación del Plan Nacional de Competitividad y Productividad Nacional promovido por el Ministerio de Economía y Finanzas (Decreto Supremo N. ${ }^{\circ} 255$ 2019-EF, 2019), siendo relevante tener en consideración el uso óptimo de la inclusión financiera como instrumento de formalización tributaria para tener un sistema tributario competitivo con una mayor presión tributaria y un Estado mejor organizado para eventos imprevistos como el último suscitado con la COVID-19, siendo una oportunidad única de reforma integral. La regulación idónea de la formalización tributaria y los créditos MYPE obtenido por personas naturales, tendría en efecto directo y positivo en el incremento de la base tributaria y en la reducción de los costos financieros para las MYPE, dado que se reduciría los riesgos crediticios al colocar préstamos a micro y pequeñas empresas formales.

Como agenda futura, es relevante considerar nuevos estudios segmentados de inclusión financiera e incumplimiento tributario por departamentos geográficos, actividad económica, nivel de ingresos y trabajadores contratados, a fin de contar con un diagnóstico efectivo de la situación de las MYPE personas naturales informales, considerando las externalidades descritas y comparando además la situación del segmento MYPE formal (personas naturales y jurídicas).

\section{REFERENCIAS}

Banco Central de Reserva del Perú. (2020). Gerencia Central de Estudios Económicos. Recuperado de: https:// estadisticas.bcrp.gob.pe/estadisticas/series/cuadros/ memoria/ca_001\%09\%09\%09\%09\%09\%09\%0A

Carballo, I. E., y Dalle-Nogare, F. (2019). Fintech and financial inclusion: the cases of Mexico, Chile, and Peru. Revista CEA, 5(10), 11-34. https://doi. org/10.22430/24223182.1441

Cardona Ruiz, D. E., Hoyos, M. C., y Saavedra-Caballero, F. (2018). Género e inclusión financiera en Colombia. Ecos de Economía, 22(46), 60-90. https://doi. org/10.17230/ecos.2018.46.3

Comisión Económica para América Latina y el Caribe. (2019). Panorama Fiscal de América Latina y el Caribe 2019: políticas tributarias para la movilización de recursos en el marco de la Agenda 2030 para el 
Desarrollo Sostenible. Panorama Fiscal de América Latina y el Caribe. Recuperado de: https://repositorio.cepal.org/bitstream/handle/11362/44516/1/ S1900075_es.pdf

Decreto Legislativo $\mathrm{N}^{\circ}$.943. (2003). Ley de Registro Único de Contribuyentes. Recuperado de: http://www.sunat. gob.pe/legislacion/ruc/fdetalle.htm

Decreto Supremo N. ${ }^{\circ} 133-2003-E F . ~(2003)$. TUO del Código Tributario. Recuperado de: http://www.sunat.gob. pe/legislacion/codigo/fdetalle.html

Decreto Supremo N. ${ }^{\circ 255-2019-E F . ~(2019) . ~ P o l i t i c a ~ N a c i o-~}$ nal de Inclusión Financiera. Recuperado de: https:// www.gob.pe/institucion/mef/normas-legales/287084255-2019-ef

Decreto Supremo N. ${ }^{\circ} 430-2020-E F . ~(2020)$. Reglamentan la información financiera que las empresas del Sistema Financiero deben suministrar a la SUNAT para el combate de la evasión y elusión tributarias. Recuperado de: https://busquedas.elperuano.pe/normaslegales/reglamentan-la-informacion financiera-que-las-empresasdel-s-decreto-supremo-n-430-2020-ef-1916565-3/

Espitia, J., Reyes, L. C., y Villabona, J. O. (2017). Sobre la reforma tributaria estructural que se requiere en Colombia. Reflexiones y propuestas, 19(36), 149-174.

Girón, A., de la Vega Shiota, V., y Vélez, D. (2018). Financial inclusion \& gender perspective: APEC Member Economies. México y La Cuenca Del Pacífico, 7(21), 27-51. https://doi.org/10.32870/mycp.v7i21.562

Goldenberg Serrano, J. L. (2020). Los dilemas de la inclusión financiera: contexto y mirada desde la realidad chilena. Revista de La Facultad de Derecho, 1180329, 1-36. https://doi.org/10.22187/rfd2020n48a9

Instituto Nacional de Estadística e Informática. (2019). Producción y empleo informal en el Perú. Recuperado de: https://www.inei.gob.pe/media/MenuRecursivo/ publicaciones_digitales/Est/Lib1701/libro.pdf

Lahura, E. (2016). Sistema financiero, informalidad y evasión tributaria en Perú. Revista Estudios Económicos, 32, 55-70. Recuperado de: www.bcrp.gob.pe/publicaciones/revista-estudios-economicos.html

Lara Rodríguez, J., Tosi Furtado, A., y Altimiras Martin, A. (2019). Minería del platino y el oro en Chocó: pobreza, riqueza natural e informalidad. Revista de Economía Institucional, 22(42), 241-268. https://doi. org/10.18601/01245996.v22n42.10
León, J. (2018). Inclusión financiera de las pequeñas y medianas empresas en Colombia. In La inclusión productiva y para la inserción financiera el papel de la banca de desarrollo. Recuperado de: https://repositorio.cepal. org/handle/11362/44233

Ministerio de Economía y Finanzas. (2018a). Compendio Tributario. Recuperado de: https://estadisticas.bcrp. gob.pe/estadisticas/series/cuadros/memoria/ca_001

Ministerio de Economía y Finanzas. (2018b). Competitividad y Productividad Consejo Nacional de Competitividad y Ministerio de Economía y Finanzas. Recuperado de: https://www.mef.gob.pe/contenidos/archivosdescarga/competitividad_productividad.pdf

Ministerio de Economía y Finanzas. (2019). Marco Macroeconómico Multianual 2020-2023. Diario Oficial El Peruano. Recuperado de: https://www.mef.gob.pe/contenidos/pol_econ/marco_macro/MMM_2020_2023]

Organización para la Cooperación y el Desarrollo Económico. (2020). Revenue Statistics in Latin America and the Caribbean 1990-2018. Publishing. https://doi.org/ https://doi.org/10.1787/68739b9b-en-es

Orazi, S., Martinez, L. B., \& Vigier, H. P. (2019). La inclusión financiera en América Latina y Europa. Ensayos de Economía, 29(55), 181-204. https://doi.org/10.15446/ ede.v29n55.79425

Superintendencia de Banca y Seguros. (2020). Reportes del Sistema Financiero. Reportes Del Siistema Financiero. Recuperado de: https://www.sbs.gob.pe/estadisticas-y-publicaciones/estadisticas-/sistema-financiero_

Superintendencia Nacional de Aduanas y de Administración Tributaria. (2020). Estadísticas y Estudios. Recuperado de: http://www.sunat.gob.pe/estadisticasestudios/registro-baseTributaria.html

Tuesta, S., y Espinoza, J. (2020). El impacto de las obligaciones tributarias electrónicas en la formalización de las micro y pequeñas empresas peruanas. Revista de Investigación y Cultura, 9(01), 33-44. Recuperado de: https://core.ac.uk/download/pdf/287243797.pdf.

Zamalloa, J. C., Obando, E. C., y Rodríguez, V. (2017). Inclusión Financiera en Distritos De Perú: Enfoque Multidimensional y Factores Determinantes. Quipukamayoc, 24(46), 111-126. https://doi.org/10.15381/quipu. v24i46.13246 